IT is now generally accepted that structures containing regions of heteroduplex DNA, with one strand of the duplex contributed from each parent molecule, are essential intermediates in the biochemically complex process of genetic recombination. During heterozygous crosses, the inclusion of a genetic marker within such a hybrid segment of DNA will mean the formation of a mismatched base-pair, or some other localised distortion of the DNA structure. It has been clearly shown in several systems that localised aberrations in DNA structure are subject to enzymic repair, by processes presumed to be analogous to the excision of pyrimidine dimers from ultravioletirradiated DNA. This repair of heteroduplex mismatches is believed to be a fundamental step in the recombination process and to be primarily responsible for the unequal segregation of the components of an allele-pair in the process of gene conversion.

Ahmad, Holloman and Holliday, in this week's issue of Nature (page 54) have now demonstrated that a nuclease from the smut fungus, Ustilago maydis, which had previously been implicated in genetic recombination, has the properties expected of an enzyme involved in the repair of mismatching heteroduplices.

The enzyme, DNase I, purified from extracts of wild-type $U$. maydis, introduces single-strand breaks in linear, duplex and DNA molecules and reduces linear, single-stranded DNA to small oligonucleotides (Holloman, J. biol. Chem., 248, 8114 ; 1973). Ahmad et al. have now studied the activity of the nuclease towards linear duplex DNA

the experiments reported by Papaioannou $e t$ al. three lines of teratocarcinoma cells cultured in vitro have been used as a source of donor cells and out of 121 animals born from inoculated blastocysts 11 showed evidence for chimaerism as judged by glucose phosphate isomerase isozyme, melanin and agouti markers. A proportion of the chimaerae from one cell line developed tumours, and some tissues (blood for example) do not appear to be chimaeric, but overall these results are very good evidence that at least some cultured teratocarcinoma cells are able to participate in normal development. As Papaioannou et al. point out, one implication of their experiment is that it should now be possible to select mutants of the cultured cells and use them to study metabolic cooperation and cell interaction during development. molecules containing local distortions, using heteroduplex molecules made from the separated strands of the DNA of bacteriophage SPP1. The state of the DNA can be sensitively monitored through its efficiency in the formation of infectious centres on transfection of competent cells of B. subtilis. Spatz and Trautner, who previously used this system to study the in vivo repair of heteroduplices (Molec. gen. Genet., 109, 84; 1970),

\section{Mismatched DNA and recombination}

from a Correspondent

have shown that a single-strand nick inactivates the DNA molecule in the transfection assay.

During a standard exposure of the DNA to the nuclease, the extent of inactivation of heteroduplices containing a single base-pair mismatch is about 6-fold greater than that of the homoduplices. Heteroduplices containing a small deletion on one strand are better substrates than those containing single base-pair mismatches. Thus the Ustilago enzyme is clearly able to recognise and preferentially attack those molecules containing distortions of the bi-helical structure. The nature of the biologically inactive products of the enzymic reaction was not investigated.

Well characterised nucleases from Neurospora crassa and Aspergillus oryzae have many similarities to the Ustilago enzyme, including the marked specificity for polynucleotides with disordered structures. The novel feature of the Ustilago enzyme is that it is strongly implicated in genetic recombination. Double mutants of Ustilago, isolated by screening for deficiencies in both extracellular and intracellular doexyribonucleases, show no detectable allelic recombination (Badman, Genet. Res., 20, 213; 1972) and much reduced DNase I activity (Holloman and Holliday, J. biol. Chem., 248, 8107-8113; 1973). The presumption that the deficiency in recombination and the loss of deoxyribonuclease activity are due to the same genetic lesions is supported by the finding that the two phenotypes are segregated together through meiosis.

The unusual specificity of these fungal nucleases makes them of more general interest as tools in molecular genetics. Their value is strikingly illustrated in a recent paper by Shenk, Rhodes, Rigby and Berg (Proc. natn. Acad. Sci. U.S.A., 72, 989-993; 1975) who have used the Aspergillus nuclease, $\mathbf{S 1}$, to analyse mutations in the genome of the monkey virus, SV40.

Shenk et al. demonstrates that the S1 nuclease specifically hydrolyses linear heteroduplices of SV40 DNA at the position of a single-strand nick, a small denaturation loop or even, in some cases, a single base mismatch. This property should have remarkable ramifications in molecular genetics, since it potentiates accurate localisation of genetic lesions in systems devoid of genetic recombination and of lesions that cause no phenotypic change. It should also facilitate the isolation of small, discrete segments of a genome in the many cases where the segment can be bounded by two suitable mutations. Satisfactory S1 nuclease is already commercially available!

\section{Evolution of tolerance to pollution from Peter D. Moore}

Although attention has recently been paid to the influence of pollutants on the structure and metabolism of whole ecosystems (for example Woodwell, Science, 618, 429; 1970), the effects of pollution on species composition have been the subject of rather more research. Detailed observational studies, such as those of Hawksworth and Rose (Nature, 227, 145; 1970) on epiphytic lichens have even made it possible to estimate ambient pollution levels from the species composition of communities. Such studies demonstrate that different species differ in their tolerance to a given pollutant, some responding at lower thresholds than others. But even within populations of a single species, the presence of a pollutant in the environment selects against those individuals which are most sensitive to it. This has been demonstrated most effectively in the evolution of heavy metal tolerance in grasses near abandoned mines (Antonovics, Bradshaw and Turner, Adv. Ecol. Res., 7, 1; 1971), but an increasing amount of evidence suggests that air pollutants are exerting a similar influence.

The classic work of Kettlewell (see The Evolution of Melanism, Clarendon Press, Oxford, 1973) on the development of melanism as a response to air pollution by particulate matter, has demonstrated that such selection occurs among insect populations. The problem can be a complex one, however, as has been shown recently in the case of the two spot ladybird (Adalia bipunctata). Creed (Nature, 249, 391; 1974) described the high incidence of melanism 